Pierre-Alain Duc, Jonathan Braine and Elias Brinks, eds.

\title{
Double Nuclei and "TDGs": Colliding or Activity of Nucleus Monster?
}

\author{
Edward Khachikian \\ Ambartsumian Byurakan Astrophysical Observatory, Armenia \\ Yervant Terzian \\ Cornell University, USA
}

\begin{abstract}
It is known that among active galaxies (AG) with strong emission lines ( UV-galaxies, Sy 1 and Sy2, Markarian and Kazarian galaxies, Radio-galaxies, QSO's host galaxies and so on) there are large per cent of objects with double and multiple nucleus. The common sizes and volumes of these nuclei are on the order of a few hundred parsecs or kilo-parsecs. In fact these are not double galaxies or clusters of galaxies as many of astronomers believe, but just the complicated nucleus of AG. The problem is: what are the nature and the birth of these objects? There are in fact two basic suppositions in the subject: (a) The complicated nuclei are the result of merging or colliding of two or more galaxies, or: (b) They are the results of nuclear activity. The results of detailed spectroscopic observations of a number of "tidal galaxies", carried out with the 5m Palomar telescope, 2.6m telescope of Ambartsumian Byurakan astrophysical observatory (multi-pupil spectroscopy with Tiger receiver) and $6 \mathrm{~m}$ telescope of Special Astrophysical observatory of Russia are presented. It is shown that in many cases the "tidal dwarf galaxies "(or actually complicated nucleus) are the result of galactic nuclear activity.
\end{abstract}

\section{Introduction}

Stemming from Ambartsumian's discovery of the phenomenon of activity of galactic nuclei given in his pioneering works (Ambartsumian, 1958, 1998), numerous attempts have been done to clear up its physical description.

Most likely that the eruptive activity of galactic nuclei is due to the violent outburst events of transformations of "superdense matter" in galaxies where the creation process is at work. As was said figuratively by Alan Sandage many years ago: "No astronomers would deny today mystery indeed surrounds the nuclei of galaxies, and Ambartsumian was the first who understood what a rich reward is contained in this treasury." Dr. Duc suggested me to remind here about the important article published by Ambartsumian in the end of 50s concerning the ejections of blue condensations from the nuclei of elliptical galaxies (Ambartsumian \& Shahbazian, 1957) which turned out to be compact dwarf galaxies after spectroscopic observations by Stockton $(1968,1972)$ and later by Arp, myself and many others. 
Ambartsumian noted the following galaxies showing this physical property: NGC 3561, IC1181, NGC 596, NGC 4061, IC 847 and some Anon galaxies. It was known that in the nuclear region of some radio-galaxies there are jets with condensations. He thought that it was possible to find galaxies with brighter and larger condensations like dwarf galaxies and he found them. Then Ambartsumian suggested his well-known idea on activity of nuclei of galaxies.

One of the forms of activity he considered the division (decay) of the nucleus of AG on two more or less equal parts (instead of forming condensations or dwarf galaxies).

A thousand galaxies with strong ultraviolet excess (UV-galaxies) have been discovered ever since in Byurakan Observatory under his direction. I was lucky to be first to observe spectra of these object in detailed and in 1968 named them "Markarian" galaxies.

Later Daniel Weedman and I (1971) discovered that $10 \%$ of these objects turned out to be Seyfert galaxies. Then we found that $10 \%$ of UV -galaxies have double nuclei (Khachikian et al. 1979). Now it is clear that so-called Sargent-Searle ( Searle \& Sargent, 1972) objects as well as the galaxies studied by Shaver and Chen (1985) also are double. I don't speak already about radiogalaxies most of which show double features in the central parts.

UV-galaxies do not form a homogeneous group of galaxies. There are quite different objects among them with different types of activity, which can be classified by spectrum in six different groups and, which morphologically span all possible types ranging from all Hubble types to Zwicky galaxies, Haro objects, BL Lacs and so on. Their investigation is an outstanding problem.

Furthermore, a remarkable surge of activity for theoretical investigation of origin of EHECR has arisen with current experiments (Hayashida et al., 1999; Watson, 2000; Bertou et al, 2000) towards an explanation of unexpected flux of particles with energies beyond the, so-called, Greizen-Zatsepin-Kuzmin (GZK) cutoff, which severely challenges all conventional source models. With next generation experiments such as the High Resolution Fly's Eye (recently started operating), the Pierre Auger Observatory whose data taking started recently, the Telescope Array, EUSO/OWL/Airwatch projects, will increase the available statistics from the HiRes and AGASA experiments by order of magnitude. To innovate the solution to vexing problems above recently a definite pattern for the theoretical description of both microscopic models of AGN and origin of EHECR from a unified viewpoint is emerged (Ter-Kazarian, 1989; 1992; 2001,Ter-Kazarian et al, 2003), which has an actual physical realization of Ambartsumian's key vision.

The guiding line is to treat the EHECR as a signature of presence of superdense "protomatter" sources in the Universe. To go any further it is entirely feasible to study as well the most interesting problems of nature of double and multi-nucleus galaxies, twin galaxies (Arp, Khachikian, Heidmann 1974; Nordgren et al. 1995), and to clear up whether this phenomenon is due to colliding and merging of galaxies or to tremendous activity of nucleus of galaxy. For these purposes the observations have been made with the $2.6 \mathrm{~m}$ telescope of BAO and the large telescopes of SAO and USA. 


\section{Objectives}

To investigate in detail the nature of multi-nucleus features of the active galaxies indicating complex geometries and the asymmetric outflow of matter and beams of non-isotropic emitted radiation. Much theoretical efforts has been, and is being, spent on hypotheses of accretion disks, captures of separate galaxies by larger and more massive objects, and in fall of matter into central massive highlycondensed objects, as a mechanism for producing the energy observed in, for example, the powerful radio lobes in radio galaxies and the energy radiated by QSOs. One of the key questions is to clarify the nature and formation of double and multiple nuclei in active galaxies.

Nowadays it seems very fashionable among the majority of scientists to talk about "mergers" of galaxies to explain their unusual morphology. Presumably the prejudice towards merging is based on the idea that gravity is the only force acting. Actually, in the cases in which tidal effects can clearly be seen to be presents, it reasonable to accept the merging hypotheses. But, the most of observational data are difficult to reconcile with the hypothesis of gravitational merging of two or many galaxies of the same type. If explosive events are clearly seen, even if we do not understand them, the possibility that objects are separating rather than merging must be considered. The available physical features of these objects speaks in favor of common physical nature of nuclei components, thus, perhaps there is other scenario of double nuclei formation by means of double proto-nuclei creation. Some typical examples of this kind of galaxies are shown below:

1. Mark.266. Double nucleus Sy2 type galaxy, both nuclei have complicated structure. Two new emission regions were discovered in this galaxy. With the four radio sources in the central part of this galaxy: two of them coincide with the optical nuclei and another two between them, it looks that we have here rather monster nuclei than colliding system (see Khachikian, 2002)!
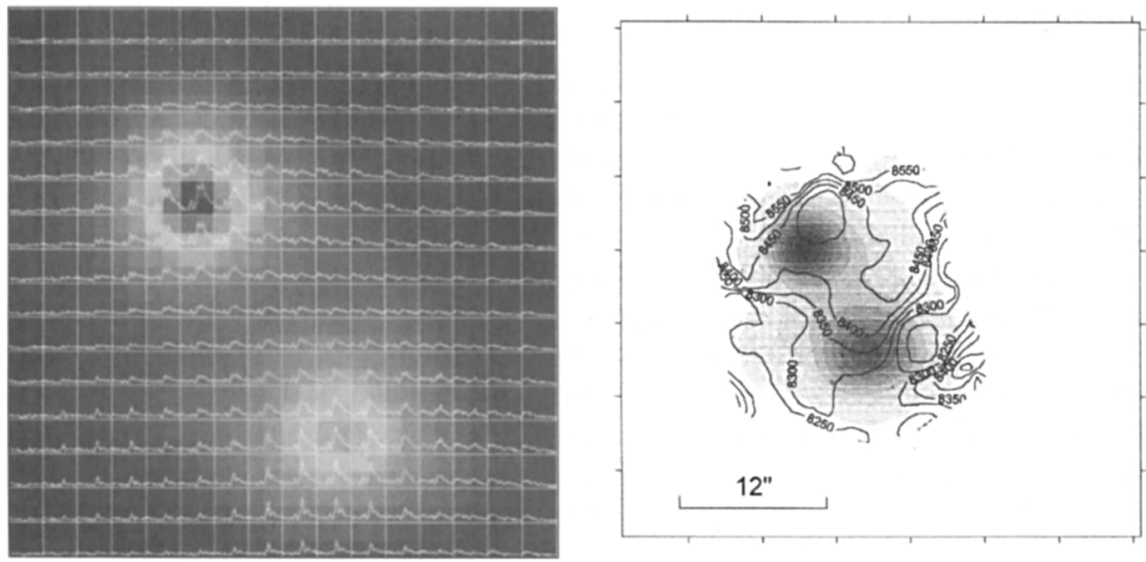

Figure 1. Mark.266 Left: profiles of $H_{\alpha}$ emission line and image in $H_{\alpha}$; Right:velocity field 
2. Mark.8. This galaxy consists of 5 bright condensations each of them is actually a super-association (SA) or giant HII region with a strong emission spectrum (Khachikian, 1972). On Fig.2 some physical characteristics of Mark.8 are presented.
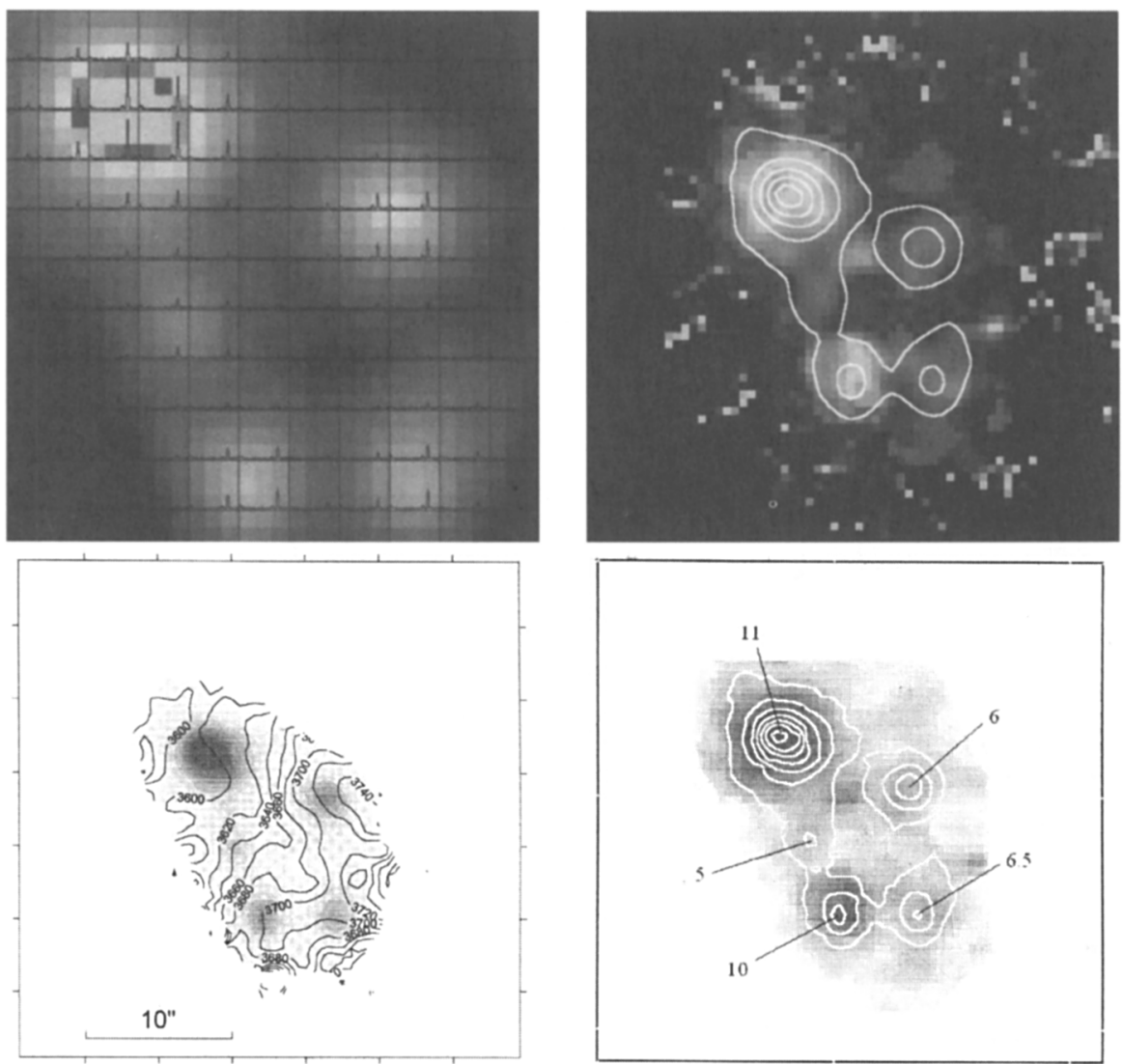

Figure 2. $\quad$ Mark.8 Top-left $H_{\alpha}$ image and profiles of $H_{\alpha}$; Top-right: [NII] image and $H_{\alpha}$ isophots; Bottom-left: velocity field; Bottom-right: $H_{\alpha} /[N I I]$ ratio

It is interesting to note that Mark.7 is very much looks like Mark8 (Khachikian, 1979; Burenkov, Khachikian, 1983). Mark.7 and Mark.8 both are single galaxies with $5 \mathrm{SA}$ and complicated cinematic structure as a result of activity of monster nucleus.

3. Twin galaxies near Mark 261 and 262 (Arp, Heidmann, Khachikian, 1974). On Fig 3 the schematic picture around Mark 261, 262, twin galaxies A and B with two "bridges" and two condensations C and D between them, as well as two objects $\mathrm{E}$ and $\mathrm{F}$ is shown. The image of twin galaxies $\mathrm{A}$ and $\mathrm{B}$ on the PSS is given on the upper left side of picture and do not differ from the stars. Except object $F$, all these objects have the same redshift and compose one physical system. The spectra of A, B, C, D and E are also shown in Fig.3. All 
these spectra are very identical with strong emission lines: HI, [OIII], [OII], [SII] and so on. Like that we have unique physical system of galaxies with the same redshift and identical spectrum and by no means can be the result of merging of 7 objects: Mark 261 and 262, A, B, C, D and E.
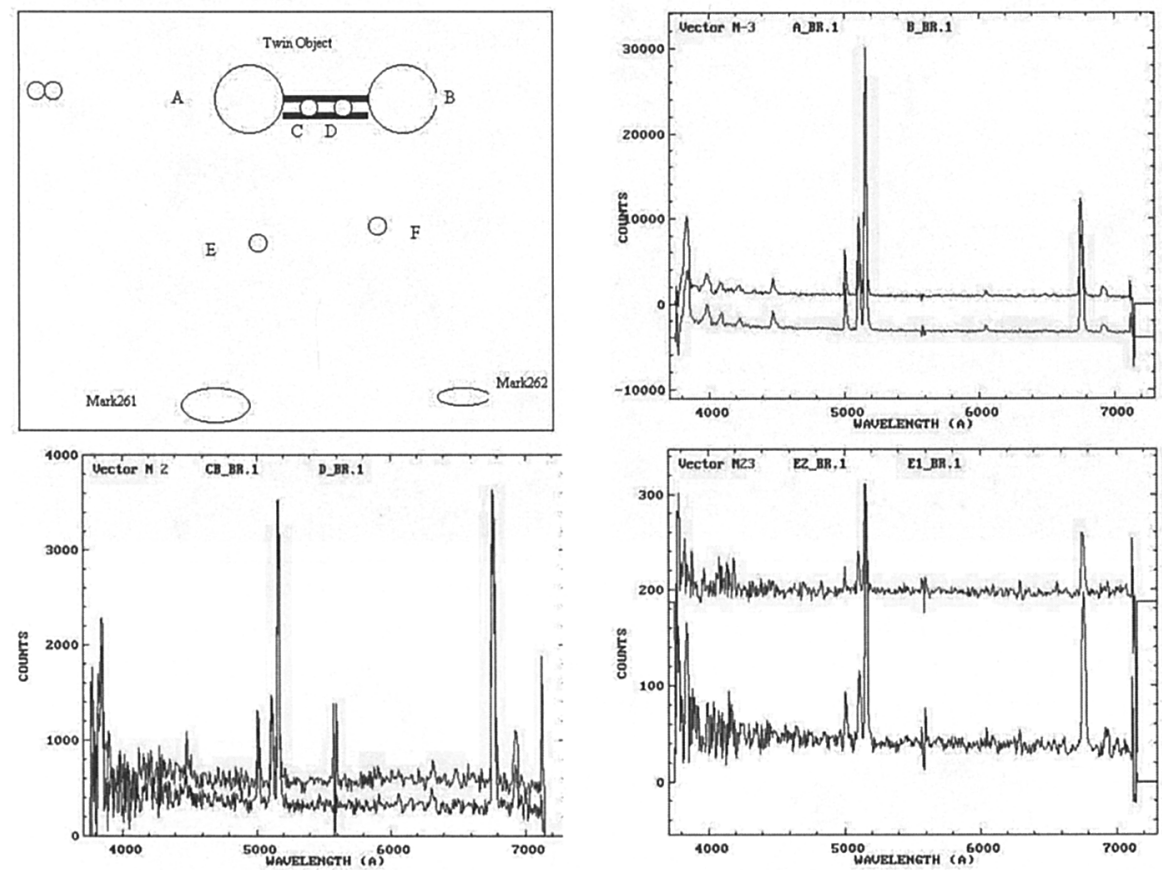

Figure 3. Top-left: Schematic picture around Mark.261 and 262; Top-right: Spectra of twin galaxies A (upper) and B; Bottom-left: Spectra of condensations C (upper) and D; Bottom-right: Spectrum of object $\mathrm{E}$

Mark.273 - is also unique galaxies with double nucleus and straight jet from southern nucleus ( Khachikian 1987; Khachikian et al.1985; Knapen et al., 1998). It is very interesting to note that the northern nucleus itself is double!

\section{Conclusion}

1. The nature of these types of galaxies can be explained with high probability by inner activity of galaxy.

2. There are a large number of double and multi nuclei galaxies, the nature of which does not speak in favor of merging or colliding galaxies.

3. The best examples of this proposition are double nucleus Seyfert galaxies (Mark 266), multi nucleus galaxies (Mark 7 and 8), Mark 273, twin galaxies. 
4. It is the time to develop the physical theory of galaxies with a complicated nucleus.

\section{References}

Ambartsumian V.A.\& Shahbazian R.K., 1957, Dokladi Academy of Sciences of Armenia , v. 25, 185.

Ambartsumian V.A.,1958, "La structure et l'evolution de l'univers", Solvey Conference, p.241, Brussels, ed. by R.Stoops.

Ambartsumian V.A., 1998, " A Life in Astrophysics ". Allerton Press, Inc. p.205-216.

Arp H., Khachikian E.Ye., Heidmann J., 1974, Astrofizika, 10, 625.

Bertou X., Boratav M., and Letessier-Selvon A., Int.J.Mod.Phys, A15 (2000) 2181-2224.

Burenkov A.N., Khachikian E.Ye., 1983, Astrofizika, 19, 826.

Hayashida N.et al., astro-ph /0008902, appendix for Astrophys.J. 522 (1999) 225 ;

Khachikian E.Ye. \& D.Weedman, 1971, Astrofizika, 7, 389.

Khachikian E.Ye., 1972, Astrofizika, 8, 529.

Khachikian E.Ye., 1979, Proceed. Symp.: Star and Stellar System, Uppsala, p107.

Khachikian E.Ye., Petrosian A.R., Sahakian K.A.,1979, Astrofizika, 15, 373.

Khachikian E,Ye. Petrosian A.R., Sahakian K.A., 1985, Astrofizika, 22, 229.

Khachikian E.Ye. 1987, Proceed. IAU Symp. No 121, Byurakan, Armenia, pp.65-79.

Khachikian E.Ye., 2002, IAU Colloquium No184, pp.3-12. ASP Conference Series, Vol.284 (Edited by R.Green, E.Khachikian, D.Sanders ).

Knapen J., et al., 1997, Ap.J., Lett., 490, L29.

Nordgren T., Helou G., Chengalur J., Khachikian E. Ye., Terzian Ye., 1995, Ap. J. Supp. Ser., 99, 461-500.

Shaver P.A. \& Chen J-S.,1985, Astron. Astrophys., 148, 443.

Searle L., Sargent W. L.W., 1972, Ap.J., 173, 25.

Stockton,A., 1968, Astron. J., 73, 887.

Stockton, A., 1972, Astrophys. J., 173, 247.

Ter-Kazarian G.T., Dokl.Akad.Nauk SSSR, 1989, 309, pp.97-101; Astrophys. \& Space Sci., 1992, 194, pp. 1-123; J. Phys.Soc. Jpn., 2001, 70, Suppl. B, pp. 84-98.

Ter-Kazarian G.T. et al. "The Universe Viewed in Gamma-Rays", 2003, Eds. R. Enomoto, M. Mori, and S. Yanagida ISSN: 0915-8502, pp.515-521, Universal Academy Press, Tokyo, Japan.

Watson A.A., Phys.Rept., 2000, 309, pp. 333-334. 